\section{Kidney \\ Blood Pressure Research}

\title{
Immediate and Late Effects of Stress on Kidneys of Prepubertal and Adult Rats
}

\author{
Roger Gaspar Marchon ${ }^{a}$ Carina Teixeira Ribeiro ${ }^{\text {Waldemar Silva Costa }}{ }^{a}$ \\ Francisco José Barcellos Sampaio ${ }^{a}$ Marco Aurélio Pereira-Sampaio a,b \\ Diogo Benchimol de Souza ${ }^{a}$
}

aUrogenital Research Unit, Rio de Janeiro State University, Rio de Janeiro, bDepartment of Morphology, Fluminense Federal University, Niteroi, Brazil

\author{
Key Words \\ Chronic Stress • Kidney • Morphometry • Rats
}

\begin{abstract}
Background/Aims: Stress had been related to physiological and morphological alterations in different organs, however kidney morphology and function after stress conditions are not well-known. To evaluate the effects of chronic stress on renal morphology in prepubertal and adult rats, and to investigate if the effects of induced stress remain stable or worsen following removal of the stress stimuli. Methods: Forty prepubertal rats (4 weeks old) and thirty six adult rats (10 weeks old) were used. Each age group was divided a into control group and a stressed group. Stressed animals were restrained in rigid tubes 2 hours per day for 6 weeks. Animals were euthanized 24 hours after the last stress stimulus for immediate evaluation (50\% of the groups), and 6 weeks after the last stress stimulus for late evaluation (50\% of the groups). Kidney weight and volume, cortical-medullar ratio, glomerular volumetric density, volume-weighted glomerular volume, and number of glomeruli per kidney were evaluated using the Image J software. Results: Prepubertal stressed animals showed reduced kidney weight and volume, and increased cortical-medullar ratio in comparison to its control group when evaluated immediately. Furthermore, stressed prepubertal and adult animals evaluated immediately had reduced glomerular volumetric density. Most importantly, all stressed groups exhibited reduced number of glomeruli per kidney. Other analyzed parameters did not difeer significantly the groups. Conclusion: Chronic stress induced before and after puberty led to irreversible glomerular loss, however, renal impairment was interrupted by removal of the stress stimuli.




\section{Kidney Blood Pressure Research}

Marchon et al.: Effects of Chronic Stress on Kidneys

\section{Introduction}

Ever since stress was described by Selye as "a syndrome produced by diverse nocuous agents", this condition has been identified in almost all societies around the globe, and may be caused by physical or psychological conditions $[1,2]$. Stress induces an organic response with increased activity in all tissues and markedly increased release of glucocorticoids, which is an attempt to respond or adapt to a new organic condition [3].

However, this organic response may lead to an acute or chronic homeostasis imbalance, and a multi-system physiological dysregulation has been described as a consequence of repeated or chronic exposure to stressors [4]. Furthermore, stress had been related not only to physiological, but also morphological alterations in different organs [5-8], including the kidney $[9,10]$.

In a previous study of our group, stereological methods were used to investigate renal morphological changes induced by immobilization stress in prepubertal rats. The study demonstrated that chronic stress before puberty causes an important reduction in the number of glomeruli [9]. Reduction in filtration surface area as consequence of glomerular loss is associated with progressive renal insufficiency and systemic hypertension $[11,12]$. Thus, the effects of chronic stress in the kidney may have serious implications, predisposing individuals to renal disease and hypertension [9].

However, the effects of chronic stress on kidney morphology and function are not wellknown, and further studies are required to clarify the role of renal morphological alterations induced by stress in the pathogenesis of renal disease. This study aims to investigate whether renal alterations are also observed in adult rats exposed to chronic stress. Furthermore, also investigated whether the alterations in renal morphology caused by stress stimuli remain stable or worsen after the removal of stress stimuli.

\section{Materials and Methods}

\section{Animals}

Seventy-six male Wistar rats were used in the experiments. The rats were kept in a temperaturecontrolled room $\left(22^{\circ} \mathrm{C} \pm 1^{\circ} \mathrm{C}\right)$ with an artificial dark-light cycle (lights on from 7:00 AM to 7:00 PM), and fed standard rat chow and water ad libitum. The Animal Care and Use Committee of the State University of Rio de Janeiro approved the handling of the animals and the study design (protocol number CEUA/007/2018).

\section{Experimental design}

The animals were divided into four groups of prepubertal rats $(n=40$; age, 4 weeks old; mean body weight, $84.8 \mathrm{~g}$ ) and four groups of adult rats ( $\mathrm{n}$ = 36; age, 10 weeks old; mean body weight, $283.4 \mathrm{~g}$ ). For each age group, one group was killed and evaluated one day after the last stress stimuli; the other groups were evaluated six weeks after the end of the stress stimuli. Additionally, for each group submitted to the stress protocol, one age-matched control group was studied. Thus, eight groups of rats were studied as follow: Group SP-I $(n=10)$, composed of prepubertal rats subjected to chronic stress and used for immediate evaluation (killed after 10 weeks of life); Group CP-I $(n=10)$, composed of prepubertal control rats killed after 10 weeks of life; Group SA-I ( $n=9$ ), composed of adult rats subjected to chronic stress and used for immediate evaluation (killed after 16 weeks of life); Group CA-I ( $\mathrm{n}=9)$, composed of adult control rats killed after 16 weeks of life; Group SP-L ( $n=10)$, composed of prepubertal rats subjected to chronic stress until 10 weeks of life and used for late evaluation (killed after 16 weeks of life); Group CP-L ( $n=10)$, composed of prepubertal control rats killed after 16 weeks of life; Group SA-L ( $n=9)$ composed of adult rats subjected to chronic stress and used for late evaluation (killed after 22 weeks of life); and Group CA-L (n=9), composed of adult control rats killed after 22 weeks of life. Fig. 1 summarizes the experimental design of the study.

Groups SP-I, CP-I, SA-I and CA-I were used to evaluate the immediate effects of stress stimuli while groups SP-L, CP-L, SA-L and CA-L were used to investigate whether the potential changes caused by stress stimuli were stable or worsened after the removal of stress stimuli. 


\section{Kidney Bloód Pressure Research}

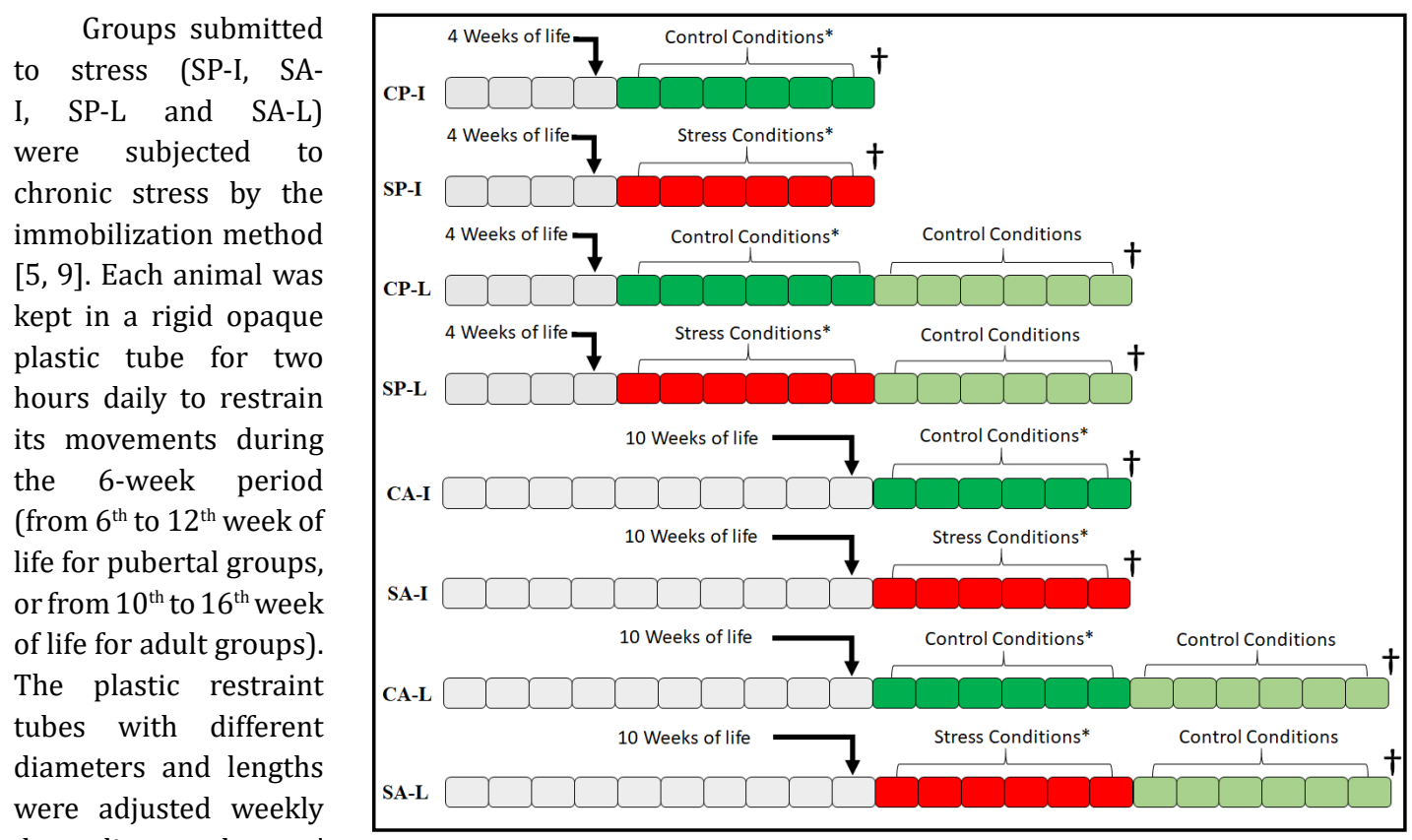

depending on the rats' size. Meanwhile, the control groups (CPI, CA-I, CP-L and CAL) were kept under normal conditions and not subjected to any stresses. Control groups, as well as stressed groups (when under the stress stimuli), were deprived of food and water for two hours per day [8].

\section{Biometrical and morphometrical analyses}

At the end of the experimental period all the animals were subjected to euthanasia, by isoflurane (Forane, Abbott Laboratories, Buenos Aires, Argentina) inhalation until cardiac arrest occurred.

Both kidneys of all animals were collected and fixed in $4 \%$ buffered formaldehyde. Kidneys were weighed and their volumes were estimated by the Scherle's method [13]. Left kidneys were transversely sliced for sequential sections of $2 \mathrm{~mm}$ thickness, which were used for determining the cortical-medullar ratio using Cavalieri's principle $[14,15]$. The absolute cortical volume (CV) was calculated by multiplying the cortical-medullary ratio by the renal volume [16].

Fragments from the right kidneys were collected and routinely processed for paraffin embedding. Sections of $5 \mu \mathrm{m}$ thickness were obtained and stained with hematoxylin and eosin. Twenty-five randomly selected histological fields of the cortex of each kidney, were analyzed. These fields were photographed with a digital camera (DP70, Olympus, Tokyo, Japan) coupled with a microscope (BX51, Olympus) under $200 \mathrm{x}$ magnification.

Glomerular volumetric density (Vv[glom]), which indicates the proportional volume occupied by the glomeruli in the cortex, was estimated by the point-counting technique with an M42 test-system $[9,17,18]$. The volume-weighted glomerular volume (VWGV), which indicates the mean volume of the glomeruli, was estimated with the point-sampled intercepts method analyzing 50 glomeruli per animal $[9,14,15]$. Analyses of $\mathrm{Vv}$ [glom] and VWGV were performed using the ImageJ software (version 1.46r, NIH, Bethesda, USA). An estimation of the total number of glomeruli per kidney was calculated by multiplying the CV by the Vv[glom] and dividing the result by the $\operatorname{VWGV}[9,16,19]$. 


\section{Kidney Blood Pressure Research}

\section{Statistical analysis}

All parameter values were analyzed using the Kolmogorov-Smirnov normality test, and all sample data were normally distributed (Gaussian distribution). The mean values in each group of rats subjected to stress stimuli were compared with those of the corresponding control group by using the one-way analysis of variance (ANOVA) with application of the Newman-Keuls post-test. All analyses were performed with the GraphPad Prism 5.0 software (GraphPad Software, San Diego, USA). Mean differences were considered significant when their $\mathrm{p}$ values were $<0.05$. All results are presented as mean \pm standard deviation.

\section{Results}

Immediate and late effects of stress stimuli on the kidneys of prepubertal rats

The kidney weight and volume of group SP-I reduced by $10.8 \%$ and $9.0 \%$, respectively, in comparison to group CP-I. These parameters were not altered among groups CP-L and SP-L.

The cortical-medullar ratio of group SP-I was 4.4\% higher than its control group, but CV was similar between these groups. No differences were observed for the cortical-medullar ratio or CV among the CP-L and SP-L groups.

Group SP-I showed reduced Vv[Glom] values in comparison to group CP-I, with a reduction of $17.4 \%$. No differences for $\mathrm{Vv}$ [Glom] were observed between the CP-L and SP-L groups. Further, no differences in VWGV were observed among the kidneys of all groups.

The total number of glomeruli per kidney was significantly smaller in stressed animals compared to its agematched controls. The kidneys of group SP-I had 20.9\% less glomeruli than those of group CP-I. Also affected by chronic stress, the SP-L group demonstrated $19.8 \%$ less glomeruli than the CP-L group. Fig. 2 illustrates the most prominent differences among immediately-evaluated groups. The morphometric data of groups CP-I, SP-I, CP-L and SP-L are presented in Table 1.

Immediate and late effects of stress stimuli on the kidneys of adult rats

Kidney weight, kidney volume, cortical-medullar ratio, and $\mathrm{CV}$ were similar between groups CA-I and SA-I, and between groups CA-L and SA-L.

The $\mathrm{Vv}$ [Glom] of group SA-I was reduced by $15.7 \%$ in comparison to group CA-I. No difference in VWGV was observed among any of the studied groups.

The total number of glomeruli per kidney was reduced in stressed adult animals which were evaluated both immediately and later. In comparison to group CA-I, group SA-I showed a glomerular reduction of $33.1 \%$. Meanwhile, group SA-L lost $16.6 \%$ of

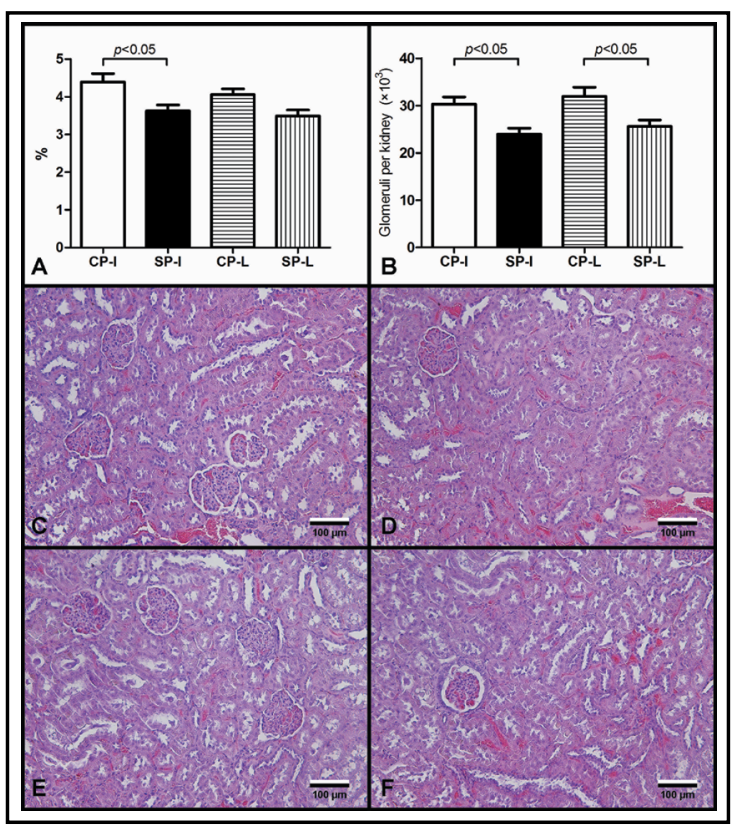

Fig. 2. Graphical representations of glomerular volumetric density (A) and total number of glomeruli per kidney (B) of prepubertal control and stressed rats immediately and lately evaluated. Images C, D, E and $F$ illustrates the renal cortex from the experimental groups: C) prepubertal control rats, evaluated immediately (CP-I); D) prepubertal stressed rats, evaluated immediately (SP-I); E) prepubertal control rats, evaluated lately (CP-L); F) prepubertal stressed rats, evaluated lately (SP-L). Hematoxylin and eosin, 200x. 


\section{Kidney Blood Pressure Research}

Table 1. Morphometric data of prepubertal rats submitted to stress conditions evaluated immediately (SPI) or lately (SP-L), and its control groups evaluated immediately (CP-I) or lately (CP-L). a: Different of CP-I. b: Different of SP-I. c: Different of CP-L

\begin{tabular}{|c|c|c|c|c|c|}
\hline Factors & CP-I & SP-I & CP-L & SP-L & $\begin{array}{c}\text { ANOVA } \\
\text { p value }\end{array}$ \\
\hline Kidney weight (g) & $1.39 \pm 0.16$ & $1.24 \pm 0.11^{\mathrm{a}}$ & $1.54 \pm 0.20^{\mathrm{a}, \mathrm{b}}$ & $1.46 \pm 0.14^{b}$ & $<0.0001$ \\
\hline Kidney volume (mL) & $1.38 \pm 0.16$ & $1.25 \pm 0.12^{\mathrm{a}}$ & $1.51 \pm 0.19 \mathrm{a}, \mathrm{b}$ & $1.44 \pm 0.14^{b}$ & $<0.0001$ \\
\hline Cortical-medullar ratio (\%) & $72.7 \pm 2.6$ & $75.9 \pm 1.8^{a}$ & $76.7 \pm 2.2^{\mathrm{a}}$ & $76.5 \pm 2.6^{\mathrm{a}}$ & 0.0013 \\
\hline Cortical Volume (mL) & $1.04 \pm 0.12$ & $0.98 \pm 0.10$ & $1.18 \pm 0.16^{\mathrm{a}, \mathrm{b}}$ & $1.11 \pm 0.13$ & 0.009 \\
\hline Vv[Glom] (\%) & $4.39 \pm 0.71$ & $3.62 \pm 0.50^{\mathrm{a}}$ & $4.06 \pm 0.46$ & $3.49 \pm 0.51^{\mathrm{a}}$ & 0.003 \\
\hline $\operatorname{VWGV}\left(\times 10^{5} \mu \mathrm{m}^{3}\right)$ & $14.96 \pm 1.30$ & $14.89 \pm 1.69$ & $15.14 \pm 1.91$ & $15.19 \pm 1.58$ & 0.972 \\
\hline Number of glomeruli per kidney $\left(\times 10^{3}\right)$ & $30.31 \pm 4.78$ & $23.97 \pm 3.93^{a}$ & $31.92 \pm 6.24 b$ & $25.60 \pm 4.26^{\mathrm{a}, \mathrm{c}}$ & 0.0021 \\
\hline
\end{tabular}

their glomeruli compared to group CAL. Fig. 3 illustrates the most prominent differences among later-evaluated groups. All morphometric data of groups CA-I, SA-I, CA-L, and SA-L are presented in Table 2.

\section{Discussion}

The present study is a sequence of a previously published manuscript, where the effects of chronic stress were investigated in prepubertal rats, immediately at the end of the stress period [9]. Groups CP-I and SP-I of the present study resembled the experimental groups of the previous study. However, the other groups in the current study addressed questions regarding the impact of stress stimuli in different phases of life (prepubertal or adult), and whether the renal impairment caused by stress stimuli is permanent, progressive, or stable.

Comparing the results of groups CP-I and SP-I to the results in a previously published study [9], many similarities and some differences were observed. In both studies, prepubertal rats submitted to stress exhibited reductions of renal weight, renal volume, $\mathrm{Vv}$ [glom], and total

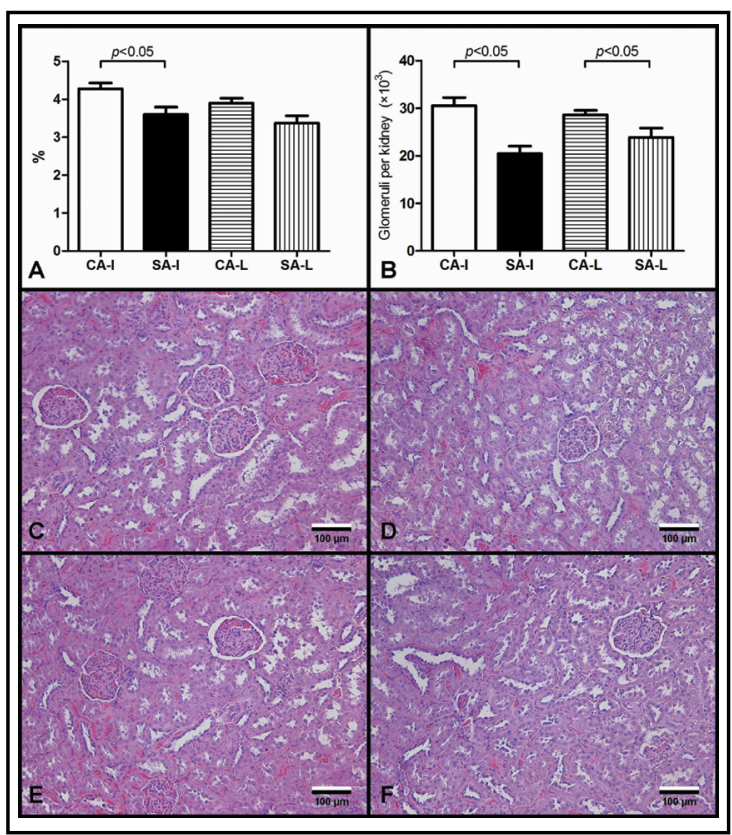

Fig. 3. Graphical representations of glomerular volumetric density (A) and total number of glomeruli per kidney (B) of adult control and stressed rats immediately and late evaluated. Images C, D, E and $F$ illustrates the renal cortex from the experimental groups: C) adult control rats, evaluated immediately (CA-I); D) adult stressed rats, evaluated immediately (SA-I); E) adult control rats, evaluated lately (CAL); F) adult stressed rats, evaluated lately (SA-L). Hematoxylin and eosin, 200x. number of glomeruli per kidney. Similarly, in both studies, no difference was found in terms of the cortical-medullar ratio and VWGV. However, these studies demonstrated different results with respect cortical volume. While Benchimol de Souza et al. [9] reported a reduction in cortical volume among stressed rats, this parameter was not affected in the SP-I groups of the present study. Moreover, the 


\section{Kidney Blood Pressure Research}

Table 2. Morphometric data of adults rats submitted to stress conditions evaluated immediately (SA-I) or lately (SA-L) and control groups evaluated immediately (CA-I) or lately (CA-L). a: Different of CA-I. b: Different of SA-I

\begin{tabular}{lccccc}
\hline Factors & CA-I & SA-I & CA-L & SA-L & $\begin{array}{r}\text { ANOVA } \\
\text { p value }\end{array}$ \\
\hline Kidney weight $(\mathrm{g})$ & $1.48 \pm 0.12$ & $1.46 \pm 0.16$ & $1.60 \pm 0.13^{\mathrm{a}, \mathrm{b}}$ & $1,51 \pm 0.12$ & 0.0213 \\
Kidney volume $(\mathrm{mL})$ & $1.50 \pm 0.12$ & $1.45 \pm 0.16$ & $1.51 \pm 0.19$ & $1.44 \pm 0.14$ & 0.5239 \\
Cortical-medullar ratio (\%) & $76.6 \pm 5.3$ & $78.0 \pm 2.4$ & $79.2 \pm 2.2$ & $80.3 \pm 1.2$ & 0.0909 \\
Cortical Volume $(\mathrm{mL})$ & $1.15 \pm 0.14$ & $1.17 \pm 0.15$ & $1.23 \pm 0.13$ & $1.22 \pm 0.13$ & 0.5443 \\
Vv[Glom] $(\%)$ & $4.27 \pm 0.45$ & $3.60 \pm 0.58^{\mathrm{a}}$ & $3.89 \pm 0.38$ & $3.36 \pm 0.59 \mathrm{a}$ & $<0.0001$ \\
VWGV $\left(\times 10^{5} \mu \mathrm{m}^{3}\right)$ & $16.24 \pm 1.69$ & $16.08 \pm 1.49$ & $17.03 \pm 1.91$ & $16.63 \pm 2.59$ & 0.3720 \\
Number of glomeruli per kidney $\left(\times 10^{3}\right)$ & $30.52 \pm 5.13$ & $20.43 \pm 4.82^{\mathrm{a}}$ & $28.58 \pm 2.92^{\mathrm{b}}$ & $23.84 \pm 5.85^{\mathrm{a}, \mathrm{c}}$ & 0.0004
\end{tabular}

morphological alterations of the aforementioned study were more severe than those of the current study. For instance, the kidney volume and total number of glomeruli per kidney in the current SP-I group were reduced by $9.0 \%$ and $20.9 \%$, respectively, compared to $31.9 \%$ and $45.0 \%$, respectively, in the previous study. Thus, the experimental designs of these studies were scrutinized. The present study had some protocol-specific differences compared to the study by Benchimol de Souza et al. [9]. For e.g., in the present study, the food and water provided to the control groups were removed for two hours per day, which was the same period for which stressed animals were restrained, also without food and water. In order to deprive the rats of food and water, for 2 hours daily, the metallic upper lid with rat chow and the water bottle was replaced by an empty lid, which generated some noise and disturbed the microenvironment. As these were the only differences in experimental design between the studies, it is possible that these procedures (both grid manipulation and food and water deprivation) were the cause of the different results between the studies.

It has been previously demonstrated that prepubertal rats had a more prolonged release of adrenocorticotropic hormone and corticosterone after acute stress in comparison to adult animals $[20,21]$. Thus, it could be assumed that adult rats are less affected by stress. However, no studies have compared the individual responses to chronic stress between prepubertal and adult rats. In the present study, several parameters were altered in prepubertal stressed rats compared to their control group, including: kidney weight, kidney volume, and corticalmedullar ratio. On the other hand, the same parameters were similar between group SA-I and its control group. Based on this fact, one could argue that adult animals were less affected by chronic stress, which would support the findings of the hypothalamic-pituitary-adrenal axis in acute stress [20,21]. Nevertheless, when evaluating the $\mathrm{Vv}$ [glom] and total number of glomeruli per kidney, it was evident that the adult animals suffered just as much drastic morphological changes, as prepubertal animals. These results support the hypothesis that chronic stress induces morphological alterations in any phase of life.

Although both prepubertal and adult stressed animals exhibited glomerular losses, glomerular reduction was not progressive following the removal of stress stimuli. Glomeruli cannot be formed or replicated after uterine life; thus, the number of glomeruli and nephrons can only decrease during life in response to kidney damage [18]. Therefore, it was reasonable for groups evaluated after a period without stress (SP-L and SA-L) to also demonstrate glomerular reduction. It is possible that the chronic stress induced would continue to cause deleterious effects and reduce the glomerular number, even after removal of the daily stress stimuli. However, the results of the SP-L and SA-L groups counter this hypothesis, and could serve as motivation for the reduction of stress. Hence, a lifestyle change that relieves, stress 


\section{Kidney \\ Blood Pressure Research}

may be recommended to stressed patients with renal disease, to avoid further glomerular loss.

Glomerular loss has been shown to be closely associated with renal disease [12, 22]. Continuous hyperfiltration by the remaining glomeruli, a normal renal adaptation, would eventually result in glomerulosclerosis and interstitial fibrosis [23]. Furthermore, the reduction in glomerular number is also associated with hypertension representing a vicious cycle where both the loss of glomeruli induces hypertension and hypertension promotes glomerular loss $[11,24,25]$. On the other hand, under stressful conditions, the hypothalamicpituitary-adrenal axis is further activated; recently, it has been demonstrated that a dominance of this axis over the renin-angiotensin-aldosterone system induce hypertension $[26,27]$. This reinforces the importance of reducing stress during any phase of life.

This study was performed in an animal model within controlled experimental conditions; therefore, our results may not accurately reflect the events in stressed human subjects. Furthermore, renal alterations may vary according to the type, intensity, and duration of stress stimulus. Thus, further research in this field is warranted.

\section{Conclusion}

The results of the present study demonstrated that chronic stress induced before or after puberty led to renal alterations, including a reduction in the number of glomeruli. White these stress-induced alterations cannot be reversed, its progression can be interrupted by removal of the stress stimuli.

\section{Acknowledgements}

This study was supported by grants from the Foundation for Research Support of Rio de Janeiro (FAPERJ), the Coordination for the Improvement of Post-Graduate Students (CAPES), and the National Council for Scientific and Technological Development (CNPq). These foundations had no involvement in the study design, data collection, analysis, and interpretation, drafting the manuscript and in the decision to submit for publication. Animal experiments conform to internationally accepted standards and have been approved by the appropriate institutional review body.

\section{Disclosure Statement}

The authors have no conflicts of interest to declare.

\section{References}

-1 Persike M, Seiffge-Krenke I: Competence in coping with stress in adolescents from three regions of the world. J Youth Adolesc 2012;41:863-879.

-2 Selye H: Syndrome produced by Diverse Nocuous Agents. Nature 1936;138:32.

$>3$ Selye $\mathrm{H}$ : The general adaptation syndrome and the diseases of adaptation. J Clin Endocrinol Metab 1946;6:117-230.

4 McEwen BS, Stellar E: Stress and the individual. Mechanisms leading to disease. Arch Intern Med 1993;153:2093-2101.

5 de Souza DB, Silva D, Cortez CM, Costa WS, Sampaio FJ: Effects of chronic stress on penile corpus cavernosum of rats. J Androl 2012;33:735-739. 


\section{Kidney \\ Blood Pressure Research}

6 Luczynski P, Moquin L, Gratton A: Chronic stress alters the dendritic morphology of callosal neurons and the acute glutamate stress response in the rat medial prefrontal cortex. Stress 2015;18:654-667.

7 Murphy MO, Cohn DM, Loria AS: Developmental origins of cardiovascular disease: Impact of early life stress in humans and rodents. Neurosci Biobehav Rev 2017;74:453-465.

8 Ribeiro CT, De Souza DB, Costa WS, Sampaio FJB, Pereira-Sampaio MA: Immediate and late effects of chronic stress in the testes of prepubertal and adult rats. Asian J Androl 2018;20:385-390.

9 Benchimol de Souza D, Silva D, Marinho Costa Silva C, Barcellos Sampaio FJ, Silva Costa W, Martins Cortez C: Effects of immobilization stress on kidneys of Wistar male rats: a morphometrical and stereological analysis. Kidney Blood Press Res 2011;34:424-429.

10 Hu Y, Gursoy E, Cardounel A, Kalimi M: Biological effects of single and repeated swimming stress in male rats: beneficial effects of glucocorticoids. Endocrine 2000;13:123-129.

11 Brenner BM, Garcia DL, Anderson S: Glomeruli and blood pressure. Less of one, more the other? Am J Hypertens 1988;1:335-347.

12 Hoy WE, Bertram JF, Denton RD, Zimanyi M, Samuel T, Hughson MD: Nephron number, glomerular volume, renal disease and hypertension. Curr Opin Nephrol Hypertens 2008;17:258-265.

13 Damasceno-Ferreira JA, Bechara GR, Costa WS, Pereira-Sampaio MA, Sampaio FJB, Souza DB: The relationship between renal warm ischemia time and glomerular loss. An experimental study in a pig model. Acta Cir Bras 2017;32:334-341.

14 Abreu L, Damasceno-Ferreira JA, Costa WS, Pereira-Sampaio MA, Sampaio FJB, de Souza DB: Glomerular Loss After Renal Radiofrequency Ablation Are Comparable to 30 Minutes of Warm Ischemia. J Endourol 2017;31:517-521.

15 Souza DB, Costa WS, Cardoso LE, Benchimol M, Pereira-Sampaio MA, Sampaio FJ: Does prolonged pneumoperitoneum affect the kidney? Oxidative stress, stereological and electron microscopy study in a rat model. Int Braz J Urol 2013;39:30-36.

-16 Assumcao RF, Pereira-Sampaio MA, Sampaio FJB, de Souza DB: Does a Ureteral Obstruction Affect the Contralateral Kidney Morphology? A Stereological Analysis in a Rodent Model. Urol Int 2018;100:327-332.

-17 Bechara GR, Damasceno-Ferreira JA, Abreu LA, Costa WS, Sampaio FJ, Pereira-Sampaio MA, de Souza DB: Glomerular Morphology and Renal Function after Warm Ischemia by Main Artery or Selective Clamping in a Porcine Model. Urol Int 2017;99:262-266.

-18 de Souza DB, de Oliveira LL, da Cruz MC, Abilio EJ, Costa WS, Pereira-Sampaio MA, Sampaio FJ: Laparoscopic partial nephrectomy under warm ischemia reduces the glomerular density in a pig model. J Endourol 2012;26:706-710.

19 Bechara GR, Damasceno-Ferreira JA, Abreu LA, Costa WS, Sampaio FJ, Pereira-Sampaio MA, Souza DB: Glomerular loss after arteriovenous and arterial clamping for renal warm ischemia in a swine model. Acta Cir Bras 2016;31:753-758.

20 Romeo RD, Lee SJ, Chhua N, McPherson CR, McEwen BS: Testosterone cannot activate an adult-like stress response in prepubertal male rats. Neuroendocrinology 2004;79:125-132.

21 Romeo RD, Lee SJ, McEwen BS: Differential stress reactivity in intact and ovariectomized prepubertal and adult female rats. Neuroendocrinology 2004;80:387-393.

-22 Puelles VG, Hoy WE, Hughson MD, Diouf B, Douglas-Denton RN, Bertram JF: Glomerular number and size variability and risk for kidney disease. Curr Opin Nephrol Hypertens 2011;20:7-15.

23 Chevalier RL: Evolution, kidney development, and chronic kidney disease. Semin Cell Dev Biol 2018; DOI:10.1016/j.semcdb.2018.05.024

-24 Hughson MD, Douglas-Denton R, Bertram JF, Hoy WE: Hypertension, glomerular number, and birth weight in African Americans and white subjects in the southeastern United States. Kidney Int 2006;69:671-678.

25 Keller G, Zimmer G, Mall G, Ritz E, Amann K: Nephron number in patients with primary hypertension. N Engl J Med 2003;348:101-108.

-26 Antonov YV, Alexandrovich YV, Redina OE, Gilinsky MA, Markel AL: Stress and hypertensive disease: adrenals as a link. Experimental study on hypertensive ISIAH rat strain. Clin Exp Hypertens 2016;38:415423.

27 Daimon M, Kamba A, Murakami H, Takahashi K, Otaka H, Makita K, Yanagimachi M, Terui K, Kageyama K, Nigawara T, Sawada K, Takahashi I, Nakaji S: Association Between Pituitary-Adrenal Axis Dominance Over the Renin-Angiotensin-Aldosterone System and Hypertension. J Clin Endocrinol Metab 2016;101:889-897. 